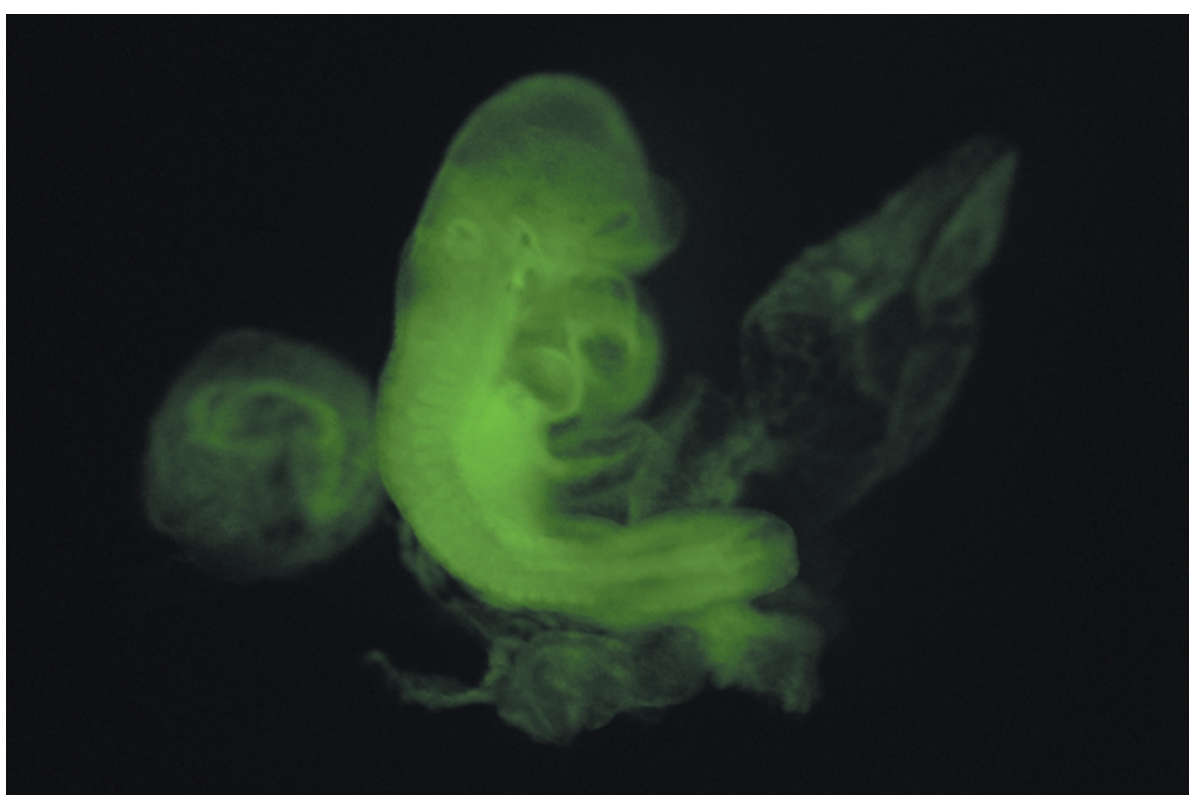

A mouse embryo injected with cells made pluripotent through stress, tagged with a fluorescent protein.

REGENERATIVE MEDICINE

\title{
Acid bath offers easy path to stem cells
}

\section{Just squeezing or bathing cells in acidic conditions can readily reprogram them into an embryonic state.}

\section{BY DAVID CYRANOSKI}

$\mathrm{I}$ n 2006, Japanese researchers reported ${ }^{1}$ a technique for creating cells that have the embryonic ability to turn into almost any cell type in the mammalian body - the nowfamous induced pluripotent stem (iPS) cells. In papers published this week in Nature, ${ }^{2,3}$, another Japanese team says that it has come up with a surprisingly simple method - exposure to stress, including a low $\mathrm{pH}$ - that can make cells that are even more malleable than iPS cells, and do it faster and more efficiently.

"It's amazing. I would have never thought external stress could have this effect," says Yoshiki Sasai, a stem-cell researcher at the RIKEN Center for Developmental Biology in Kobe, Japan, and a co-author of the latest studies. It took Haruko Obokata, a young stem-cell biologist at the same centre, five years to develop the method and persuade Sasai and others that it works. "Everyone said it was an artefact there were some really hard days," says Obokata.

Obokata says that the idea that stressing cells might make them pluripotent came to her when she was culturing cells and noticed that some, after being squeezed through a capillary tube, would shrink to a size similar to that of stem cells. She decided to try applying different kinds of stress, including heat, starvation and a high-calcium environment. Three stressors - a bacterial toxin that perforates the cell membrane, exposure to low $\mathrm{pH}$ and physical squeezing - were each able to coax the cells to show markers of pluripotency.

But to earn the name pluripotent, the cells had to show that they could turn into all cell types - demonstrated by injecting fluorescently tagged cells into a mouse embryo. If the introduced cells are pluripotent, the glowing cells show up in every tissue of the resultant mouse. This test proved tricky and required a change in strategy. Hundreds of mice made with help from mouse-cloning pioneer Teruhiko Wakayama at the University of Yamanashi, Japan, were only faintly fluorescent. Wakayama, who had initially thought that the project would probably be a "huge effort in vain", suggested stressing fully differentiated cells from newborn mice instead of those from adult mice. This worked to produce a fully green mouse embryo.

Still, the whole idea was radical, and Obokata's hope that glowing mice would be enough to win acceptance was optimistic. Her manuscript was rejected multiple times, she says.

To convince sceptics, Obokata had to prove that the pluripotent cells were converted mature cells and not pre-existing pluripotent cells. So she made pluripotent cells by stressing T cells, a type of white blood cell whose maturity is clear from a rearrangement that its genes undergo during development. She also caught the conversion of T cells to pluripotent cells on video. Obokata called the phenomenon stimulustriggered acquisition of pluripotency (STAP).

The results could fuel a long-running debate. For years, various groups of scientists have reported finding pluripotent cells in the mammalian body, such as the multipotent adult progenitor cells described ${ }^{4}$ by Catherine Verfaillie, a molecular biologist then at the University of Minnesota in Minneapolis. But others have had difficulty reproducing such findings. Obokata started the current project in the laboratory of tissue engineer Charles Vacanti at Harvard University in Cambridge, Massachusetts, by looking at cells that Vacanti's group thought to be pluripotent cells isolated from the body ${ }^{5}$. But her results suggested a different explanation: that pluripotent cells are created when the body's cells endure physical stress. "The generation of these cells is essentially Mother Nature's way of responding to injury," says Vacanti, a co-author of the latest papers ${ }^{2,3}$.

One of the most surprising findings is that the STAP cells can also form placental tissue, something that neither iPS cells nor embryonic stem cells can do. That could make cloning dramatically easier, says Wakayama. Currently, cloning requires extraction of unfertilized eggs, transfer of a donor nucleus into the egg, in vitro cultivation of an embryo and then transfer of the embryo to a surrogate. If STAP cells can create their own placenta, they could be transferred directly to the surrogate. Wakayama is cautious, however, saying that the idea is currently at "dream stage".

Obokata has already reprogrammed a dozen cell types, including those from the brain, skin, lung and liver, hinting that the method will work with most, if not all, cell types. On average, she says, $25 \%$ of the cells survive the stress and 30\% of those convert to pluripotent cells - already a higher proportion than the roughly $1 \%$ conversion rate of iPS cells, which take several weeks to become pluripotent. She now wants to use these results to examine how reprogramming in the body is related to the activity of stem cells. Obokata is also trying to make the method work with cells from adult mice and humans.

"The findings are important to understand nuclear reprogramming," says Shinya Yamanaka, who pioneered iPS cell research. "From a practical point of view toward clinical applications, I see this as a new approach to generate iPS-like cells." -

1. Takahashi, K. \& Yamanaka, S. Cell 126, 663-676 (2006).

2. Obokata, H. et al. Nature $505,641-647$ (2014)

3. Obokata, H. et al. Nature 505, 676-680 (2014).

4. Jiang, Y. et al. Nature 418, 41-49 (2002).

5. Obokata, H. et al. Tissue Eng. Part A 17, 607-615 (2011). 